\title{
Nuove evidenze di correlazione tra l'attenuazione macrosismica ed i campi di lineamenti strutturali nell'Italia meridionale
}

\author{
(New evidences of correlation between the fields of structural \\ lineaments and the macroseismic attenuation in Southern Italy)
}

\author{
A. BotTari* - B. Federico* - E. Lo GiUdice* *
}

Ricevuta il 20 Novembre, 1981

\section{Abstract}

The analysis of a large number of macroseismic fields of past and recent earthquakes in Southern Italy allows a wider check of a previous interpretative model of the anisotropy of the regional macroseismic field, in the context of a more recent point of view of the tectonics.

Our model is based on the hypothesis and analysis of the correlation between the fields of structural lineaments, characterized by the frequence and cumulative length, and the attenuation of the macroseismic intensity. We studied the physical and geometric factors which control the propagation of the seismic waves which determine the macroseismic intensity in a given place, and then considered the use of the filtre carried out by the "warp" formed by the "Giant Craks".

The Irpinia earthquake on $23^{\text {rd }}$ November 1980 is suitable for a general study of the postulated correlation, due to the extent of its macroseimic field and the different structural characteristics of the areas concerned.

\section{Riassunto}

L'analisi di un congruo numero di campi macrosismici di terremoti storici e recenti dell'Italia meridionale, consente, nel contesto di una più attuale visione della tettonica, una più ampia verifica di un precedente modello interpretativo del-

* Istituto Geofisico e Geodetico dell'Università di Messina, Messina (Italy).

** Istituto Internazionale di Vulcanologia, C.N.R., Catania (Italy). 
l'anisotropia del campo macrosismico regionale. Il modello è basato sulla correlazione, postulata e verificata, tra i campi di lineamenti strutturali, caratterizzati mediante la frequenza e la lunghezza cumulativa, e l'andamento dell'attenuazione dell'intensità macrosismica.

Rilevata l'importanza dei fattori, fisici e geometrici, che controllano la propagazione delle onde sismiche determinanti l'intensità macrosismica in un dato sito, viene presa in considerazione la funzione di filtro espletata dallo "ordito" degli sciami di Giant Cracks.

Il terremoto dell'Irpinia del 23 Novembre 1980, per l'estensione del campo macrosismico e le differenti caratteristiche strutturali delle aree interessate, si presta bene ad una generale verifica della correlazione postulata.

\section{Premessa}

L'analisi dei campi macrosismici dei terremoti storici e recenti della regione dell'Arco calabro-peloritano mette in evidenza la ricorrente differente orientazione della direzione di allungamento della prima isosista rispetto alle successive. Relativamente alle isosiste di grado più elevato (la $1^{\text {a }}$ o la $1^{\text {a }}$ e la $2^{\text {a }}$ ) è ben noto che la loro geometria dipende dai parametri della sorgente sismica (S Hebalin, 1972, 1974b). Generalmente si ritiene invece che la forma del campo macrosismico regionale, esterno al near field, essendo dipendente principalmente dalla propagazione dell'energia sismica risulti controllata dai parametri elastici del mezzo.

Lo studio delle anomalie regionali risulta di particolare interesse e pertanto sono stati proposti modelli semplificati basati sull'andamento dell'attenuazione dell'intensità macrosismica. Tale grandezza, determinata rispetto alle direzioni più salienti del campo è stata inizialmente dedotta compatibilmente con un modello di campo "normale" isotropo quale quello di Blake (BotTari et al., 1979).

Nella presente nota ci si propone una più accurata analisi dell'anisotropia dei campi macrosismici dell'Italia meridionale anche nel quadro di una più attuale visione della tettonica regionale. 


\section{RAPPRESENTAZIONE DELL'ATTENUAZIONE AZIMUTALE DELL'INTENSITA} MACROSISMICA

La caratterizzazione del campo macrosismico isotropo è largamente trattata nella letteratura recente e meno recente (B LAKE, 1941; S ponheuer, 1960; S hebalin, 1972, 1974a, 1974b; Bottari et al., 1979). In particolare il problema è stato affrontato dai presenti autori nella nota citata, riguardante la ottimizzazione di una metodologia che risultasse ben adatta allo studio dei terremoti dell'Arco calabro-peloritano. Si rimanda pertanto alla nota specificata per quanto concerne la caratterizzazione dei parametri del campo macrosismico, nella assunzione semplificatrice di isotropia dello stesso. D'altra parte la notevole anisotropia azimutale dei campi macrosismici è una circostanza ricorrente per i terremoti dell'Italia meridionale. Si rende pertanto necessaria la caratterizzazione di questa al di fuori dei limiti imposti dall'isotropia del modello di Blake, anche per una più corretta interpretazione delle anomalie regionali.

Si rileva, d'altra parte, che l'intensità $I$ in funzione della distanza $\Delta_{z}$ lungo la direzione $z$, mostra sistematicamente andamento esponenziale, sia nel caso di isotropia che di anisotropia del campo macrosismico. Ciò induce a rilevare la compatibilità fra la distribuzione dei valori di $I$ e $\triangle_{z}$ e la equazione

$$
\frac{\mathrm{d} I}{\mathrm{~d} \triangle_{z}}=-\alpha, I_{0}
$$

Quest'ultima, in termini finiti si traduce nella relazione funzionale

$$
I=I_{o} \mathbf{e}-\alpha_{\mathbf{z}} \Delta_{\mathbf{z}},
$$

ove $I_{0}$ è l'intensità macrosismica massima e $\alpha_{z}$ il coefficiente di at tenuazione nella direzione azimutale considerata. Pertanto la [2], compatibile con il modello di Blake, essendo verificata direttamente dai dati macrosismici rilevati prescinde dall'adozione di un qualunque modello, peraltro necessario per la determinazione dei parametri focali (BotTaRi et al., 1981b). In tal modo sono 
caratterizzabili valori di $\alpha_{z}$ e del suo reciproco $P_{z}$, denominato conducibilità.

Al fine di facilitare il confronto della conducibilità $P_{z}$ con altre grandezze dipendenti dall'azimut, conviene rappresentare tale parametro in diagramma stellare. Considerate le peculiari caratteristiche dei campi macrosismici si è scelto per i diagrammi stellari di $\rho_{z}$ un settore angolare di campionatura di $22^{\circ} .5$ (fig. 1).

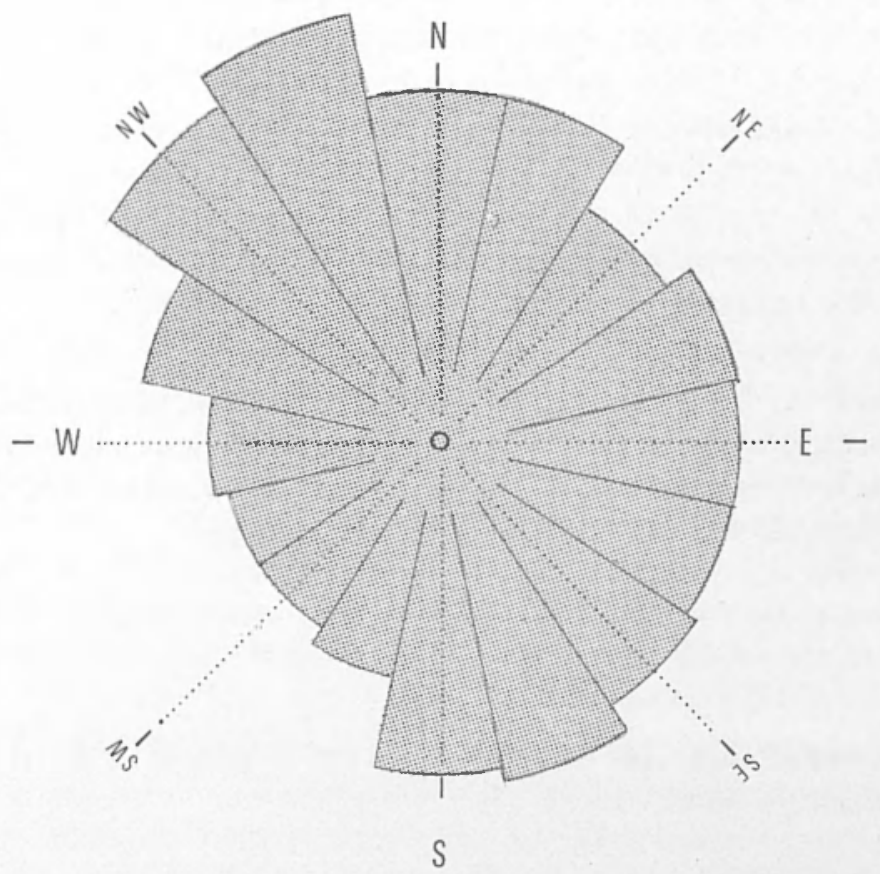

Fig. 1 - Terremoto irpino del 23 novembre 1980: diagramma stellare di $P_{2}=\alpha_{\mathrm{z}}{ }^{-1}$ relativo all'intervallo di isosiste VIII-IV e per 16 settori angolari di $22^{\circ} .5$. I valori sono normalizzati rispetto al valore massimo 471 determinato per la direttrice NNW. 
TABELLA 1

VALORI DELLA a CONDUCIBILITÁ MACROSISMICA * PER ALCUNI TERREMOTI DELL'ITALIA MERIDIONALE

\begin{tabular}{|c|c|c|c|c|c|c|}
\hline $\mathbf{N}$. & & & Terremoto & Fonte bibliogrfica & $P_{M}$ & $P_{m}$ \\
\hline 05 & 05 & FEB & 1783, Bacino di Petrace & Mercalli, 1897 & $196(240)$ & 87 (135) \\
\hline 09 & 28 & MAR & 1783, Graben di Catanzaro & ” & $137(220)$ & $133(120)$ \\
\hline 10 & 09 & MAR & 1786, Tindari & Non pubbl., AA presenti & $102(60)$ & $64(330)$ \\
\hline 16 & 28 & NOV & 1869, Monteleone & Bottari et al., 1979 & $170(40)$ & $60(290)$ \\
\hline 17 & 04 & OTT & 1870, Mangone & $" \quad "$ & $90(33)$ & $69(290)$ \\
\hline 19 & 03 & DIC & 1887. Bisignano & $" \quad " \quad$ & $208(225)$ & $119(130)$ \\
\hline 27 & 22 & APR & 1893, Montalbano d'Elicona & " & $108(50)$ & $86(245)$ \\
\hline 28 & 23 & APR & 1893, Moio Alcantara & 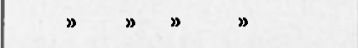 & $189(35)$ & - \\
\hline 34 & 09 & DIC & 1894, Melicuccà & Iaccarino, 1968 & $70(35)$ & $42(135)$ \\
\hline 49 & 04 & DIC & 1908, Galati Mamertino & Bottari et al., 1979 & $68(140)$ & $40(250)$ \\
\hline 50 & 10 & DIC & 1908, Montalbano d'Elicone & $\$ \gg$ & $107(40)$ & $57(135)$ \\
\hline 51 & 28 & DIC & 1908, Messina & Baratta, 1910 & $242(45)$ & $216(340)$ \\
\hline 69 & 16 & GEN & 1975, Reggio Calabria & Bottari e Lo Giudice, 1975 & $62(40)$ & $41(260)$ \\
\hline 70 & 11 & MAR & 1978, Ferruzzano & Bottari et al., 1981 a & $160(25)$ & $111(120)$ \\
\hline 71 & 15 & APR & 1978, Golfo di Patti & Barbano et al., 1979 & $173(270)$ & $107(330)$ \\
\hline 72 & 23 & NOV & 1980, Irpinia & Bottari et al., $1981 \mathrm{~b}$ & $471(315)$ & $191(225)$ \\
\hline
\end{tabular}

Nota: i valori in parentesi indicano gli azimut delle direzioni di massima $\left(P_{M}\right)$ e minima $\left(P_{m}\right)$ "conducibilità macrosismica " rappresentati in Fig. 2.

\section{A TTENUAZIONE DELL'INTENSITA MACROSISMICA NELL'ITALIA MERIDIONALE}

La direzione di allungamento dell'area mesosismica ed i valori massimi e minimi della conducibilità p., caratterizzanti i campi macrosismici di un certo numero di terremoti dell'Arco calabropeloritano, sono riportati in tabella 1 e rappresentati in Fig. 2. Tali valori sono in massima parte dedotti da alcune analisi effettuate dagli stessi autori relativamente sia a campi macrosismici di eventi storici sia ad indagini dirette su terremoti recenti. Solamente in quattro casi ci si è avvalsi acriticamente di campi macrosi- 


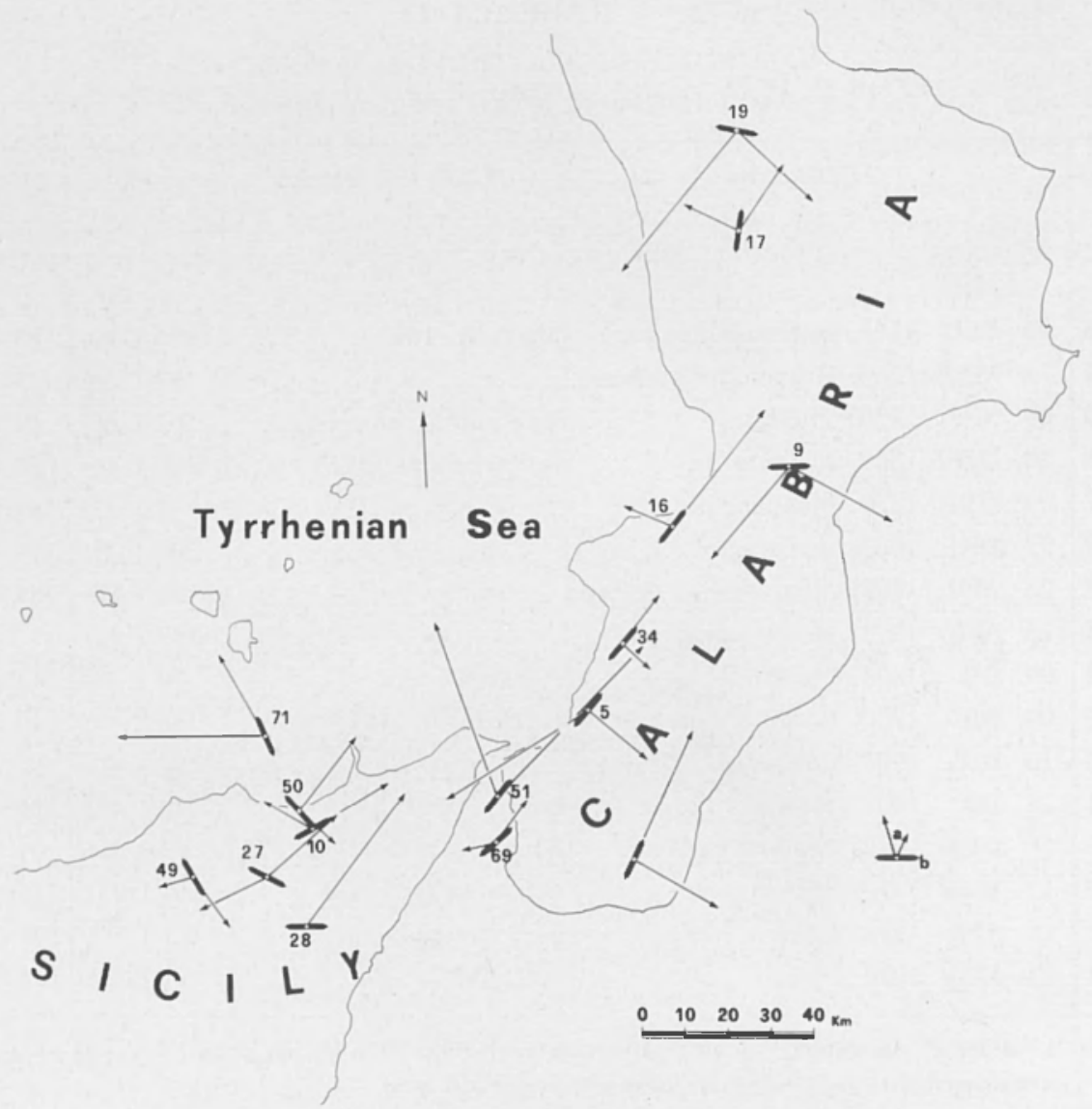

Fig. 2 - Attenuazione dell'intensità sismica lungo l'Arco: la doppia freccia $b$ indica la direzione di elongazione dell'area mesosismica; i vettori a esprimono in modulo e direzione la massima e minima « conducibilità " sismica del campo; la numerazione è relativa alla Tabella 1.

smici riportati nella letteratura sismologica (N. 34, MerCALLI, 1898; N. 5, 9, 51 B aratta, 1909).

La distribuzione degli epicentri lungo l'Arco (Fig. 2), presenta addensamenti legati verosimilmente ad aree sismogenetiche ancora non ben definite. 
Nella Sicilia nord-orientale si osserva un addensamento tra le linee Tindari-Letojanni e Naso-Floresta (Bottari et al., 1981b). L'allungamento delle prime isosiste mostra direzioni preferenziali NNW-SSE ad eccezione per i terremoti N. 10 e N. 28. Per il primo evento l'insufficienza dei dati rende estremamente incerta la definizione della forma dell'area mesosismica; mentre il secondo evento si è verificato sul versante meridionale dei Mt. Peloritani ed è caratterizzato da un quadro strutturale locale diverso (Lo GIUDICE et al., 1982). Per quanto concerne la propagazione, le direzioni preferenziali sono generalmente discordanti da quelle di allungamento delle aree mesosismiche. Precisamente per i terremoti più settentrionali si osserva una direzione di massima propagazione pressoché parallela alla costa tirrenica, cioè ENE ed $W$. Per $\mathrm{i}$ terremoti più meridionali si osserva invece una direzione di massima propagazione parallela alla costa ionica (NNE-SSW).

L'area dello Stretto di Messina e della Calabria meridionale, fino a Palmi è caratterizzata da una stretta concordanza fra l'allungamento dell'area mesosismica e la direzione di propagazione massima. Quest'ultima presenta bassa dispersione angolare e verso preferenziale in direzione $30-35^{\circ} \mathrm{E}$. Ad eccezione del terremoto calabro-messinese del 1908 (N. 51), inoltre, tutti i campi macrosismici presentano un forte assorbimento in direzione ortogonale, cioè verso SE. Caratteristiche analoghe mostrano i campi macrosismici della penisola di Capo Vaticano.

I campi della Calabria settentrionale, anche se non numerosi, presentano invece caratteristiche differenti. Le prime isosiste hanno sviluppo N-S nell'area dell'alto Crati (Sila) e presaoché E-W a Sud (Stretta di Catanzaro) ed a Nord della Sila. Pur restando quindi invariata la direzione di propagazione preferenziale manca la concordanza fra questa e quella dell'allungamento dell'area mesosismica; risalta poi l'inversione del verso di massima propagazione in corrispondenza alla direzione di allungamento E-W dell'area mesosismica. La forte attenuazione dell'intensità nella direzione SE è rilevabile anche nei campi macrosismici della Calabria settentrionale.

Da quanto esposto emerge un quadro più coerente per la Calabria che per la Sicilia, soprattutto per quanto concerne la sistematicità e la bassa dispersione angolare della direzione pre- 
ferenziale di propagazione nonché per la sua frequente coincidenza con quella di allungamento della prima isosista. L'area della Sicilia nord-orientale, essendo meno estesa mostra invece risentire maggiormente della tettonica delle aree limitrofe.

Più a Nord, nella regione campano-lucana, come mostrano i campi macrosismici di numerosi terremoti storici (Tabella 2) è costante la coerenza fra l'allungamento dell'area mesosismica in direzione appenninica (NW-SE) e la propagazione preferenziale nel campo esterno. Per alcuni terremoti $(1732,1857$ e 1930) si osserva inoltre in corrispondenza alla penisola sorrentina una propagazione preferenziale secondaria in direzione antiappenninica (SW). Ciò è confermato, e con maggiore dettaglio, dallo studio del campo macrosismico del terremoto irpino del 23 Novembre 1980, che mostra coincidenza fra la direzione di allungamento dell'area mesosismica $\left(I_{0}=\right.$ IX) con la direzione di massima propagazione (NW-SE). Ciò è rilevabile sino all'isosista di VI, a partire dalla quale si manifestano differenti direzioni preferenziali ed esattamente NNW a settentrione e SSE lungo l'Arco calabro-peloritano, nella parte meridionale del campo.

TABELLA 2

Terremoti storici nella Regione Campano-Lucana

\begin{tabular}{|c|c|c|c|c|c|}
\hline 1456, & & Dicembre & Campania, & XI & Baratta, 1901 \\
\hline 1688 , & & Giugno & Sannio & $\mathbf{x}$ & Serva, 1981 a \\
\hline 1694, & 8 & Settembre & Irpinia & $\mathbf{x}$ & Serva, 1981b \\
\hline 1732. & 29 & Novembre & Avellino & $\mathbf{x}$ & Baratta, 1901 \\
\hline 1857, & 16 & Dicembre & Basilicata & $\mathrm{x}$ & Branno et al., 1982 \\
\hline 1930, & 23 & Luglio & Irpinia & $\mathbf{x}$ & Marcelli et al., 1979 \\
\hline 1980. & 23 & Novembre & Irpinia & $\mathrm{x}$ & Bottari et al., 1981 b \\
\hline
\end{tabular}

Correlazione tra campo macrosismico e Quadro strutturale

L'Italia meridionale è interessata da sistemi strutturali longitudinali e trasversali rispetto alla catena appenninica, detti appunto appenninici e antiappenninici. Questi ultimi nella regione dell'Arco calabro-peloritano costituiscono zone di cerniera. 
In una precedente nota (Bottari et al., in press) al fine di correlare i dati macrosismici alla tettonica regionale si è utilizzato prevalentemente il campo totale dei lineamenti rilevato con il metodo Shadow (WISE, 1969) e con l'ausilio di immagini da satellite. Non ci soffermiamo, quindi, sui motivi di tale scelta ma notiamo che dovendo qui trattare un'area più estesa della precedente, l'omogeneità dell'approccio statistico e della campionatura areale, caratteristica di tale metodologia è ancora più necessaria. Ricordiamo inoltre che per la presente analisi risultano d'importanza pressoché irrilevante le limitazioni del metodo di Wise connesse alla scelta della scala, alla risoluzione del plastico ed alla mancanza di informazioni circa i lineamenti non morfologici.

L'analisi della distribuzione dei lineamenti morfologici secondo il metodo Shadow è stata condotta su scala $1: 1.000 .000$ su aree di $50 \mathrm{Km}$ di raggio, centrate nei nodi di un reticolo a maglie aventi ampiezza di un grado di latitudine e longitudine rispettivamente. La frequenza dei lineamenti e la distribuzione per lunghezza cumulativa sono rappresentate in diagrammi stellari. Nelle figure 3 e 4 sono riportati i diagrammi relativi all'Italia meridionale e da questi possono ricavarsi i domini dei lineamenti rilevati nell'area dell'Arco calabro-peloritano e dell'Appennino meridionale (WISE et al., 1979).

Il dominio $20^{\circ} \mathrm{E}$ è presente (Fig. 3) nell'Aspromonte, nelle Serre e - con un forte picco - nella Sicilia Nord-orientale. In tutta la Calabria è prevalente il dominio $10^{\circ} \mathrm{E}$ e si osserva anche il dominio $20^{\circ} \mathrm{W}$; quello $15^{\circ} \mathrm{W}$ appare invece nella Sicilia Nordorientale. Sempre nel quadrante occidentale, il dominio $35^{\circ} \mathrm{W}$ è rilevabile con un picco moderato solamente in Sicilia e quello $60^{\circ} \mathrm{W}$ in Calabria nel Golfo di Squillace e nella Sila Grande. Infine sono individuabili in Sicilia i domini $75^{\circ} \mathrm{W}$ e $75^{\circ} \mathrm{E}$, ed in tutta la Calabria settentrionale il dominio E-W.

Nella regione campano-lucana (Fig. 4) i domini di lineamenti mostrano in modo più tipico î loro carattere appenninico o antiappenninico. Tra i primi notiamo nella parte settentrionale e più spiccatamente in Irpinia il dominio $\mathrm{N} 45^{\circ} \mathrm{W}$ a cui si sovrappone distintamente, e con prevalenza nettamente maggiore nella parte meridionale, il dominio $\mathrm{N} 60^{\circ} \mathrm{W}$. Al primo dominio $\left(\mathrm{N} 45^{\circ} \mathrm{W}\right)$ che caratterizza particolarmente l'Appennino umbro sono riferibili 


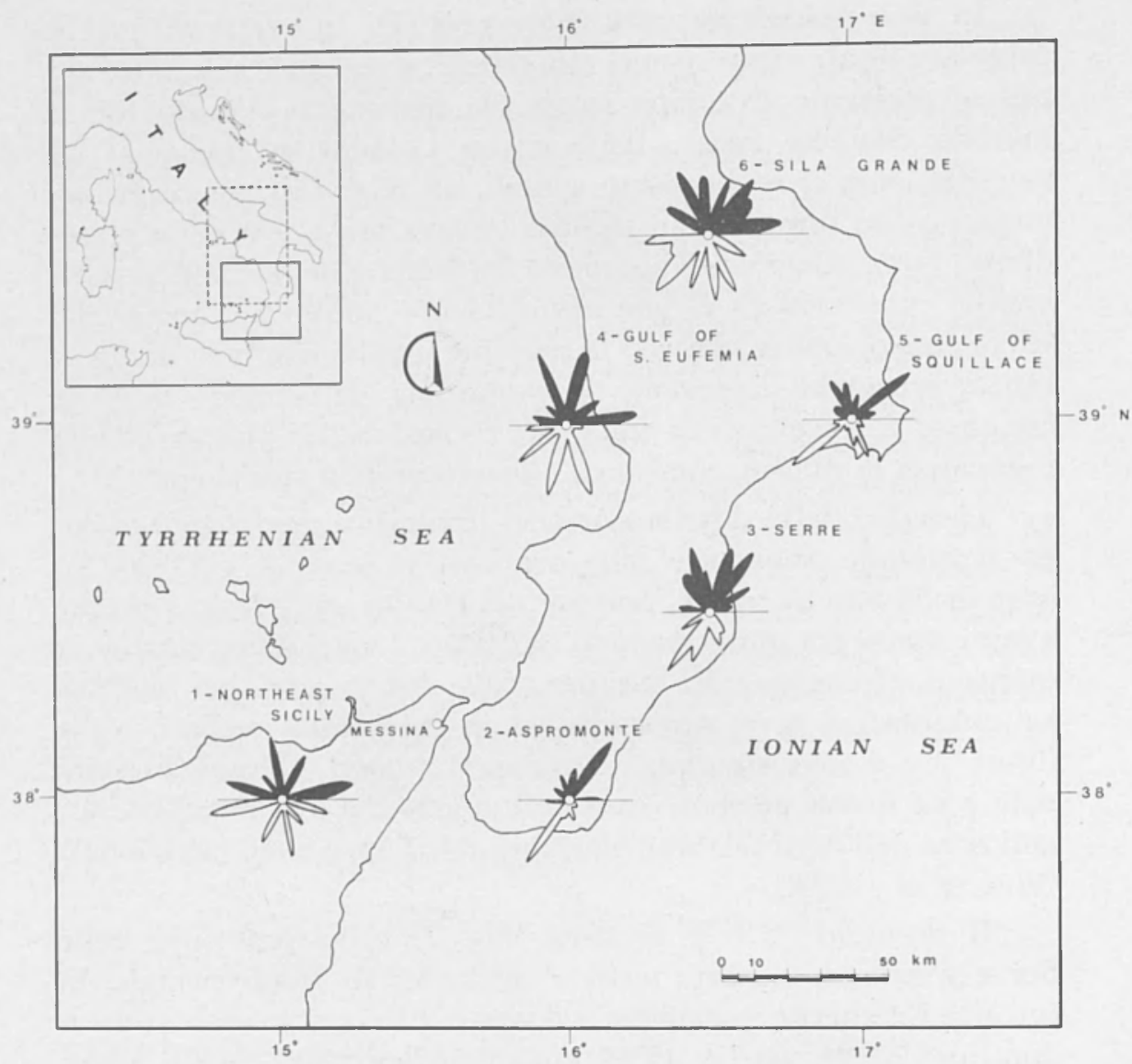

Fig. 3 - Distribuzione dei lineamenti morfologici rilevati con il metodo "Shadow " nella regione dell'Arco calabro-peloritano (da Wise et al., 1979). La parte superiore dei diagrammi (in nero) è relativa alla frequenza dei lineamenti; la parte inferiore (in bianco) alle lunghezze cumulative degli stessi.

sistemi di faglie e graben di recente formazione osservabili sia su immagini da satellite che sul terreno. Vari Autori (C APuTo e PostPischl, 1974; OGninen et al., 1975; WISE et al., 1979) rilevano una forte correlazione spaziale e forse anche geometrica tra questo dominio e l'attività sismica attuale. Il secondo dominio $\left(\mathrm{N} 60^{\circ} \mathrm{W}\right)$ caratterizza particolarmente la fossa bradanica, a Sud di Bari, e 


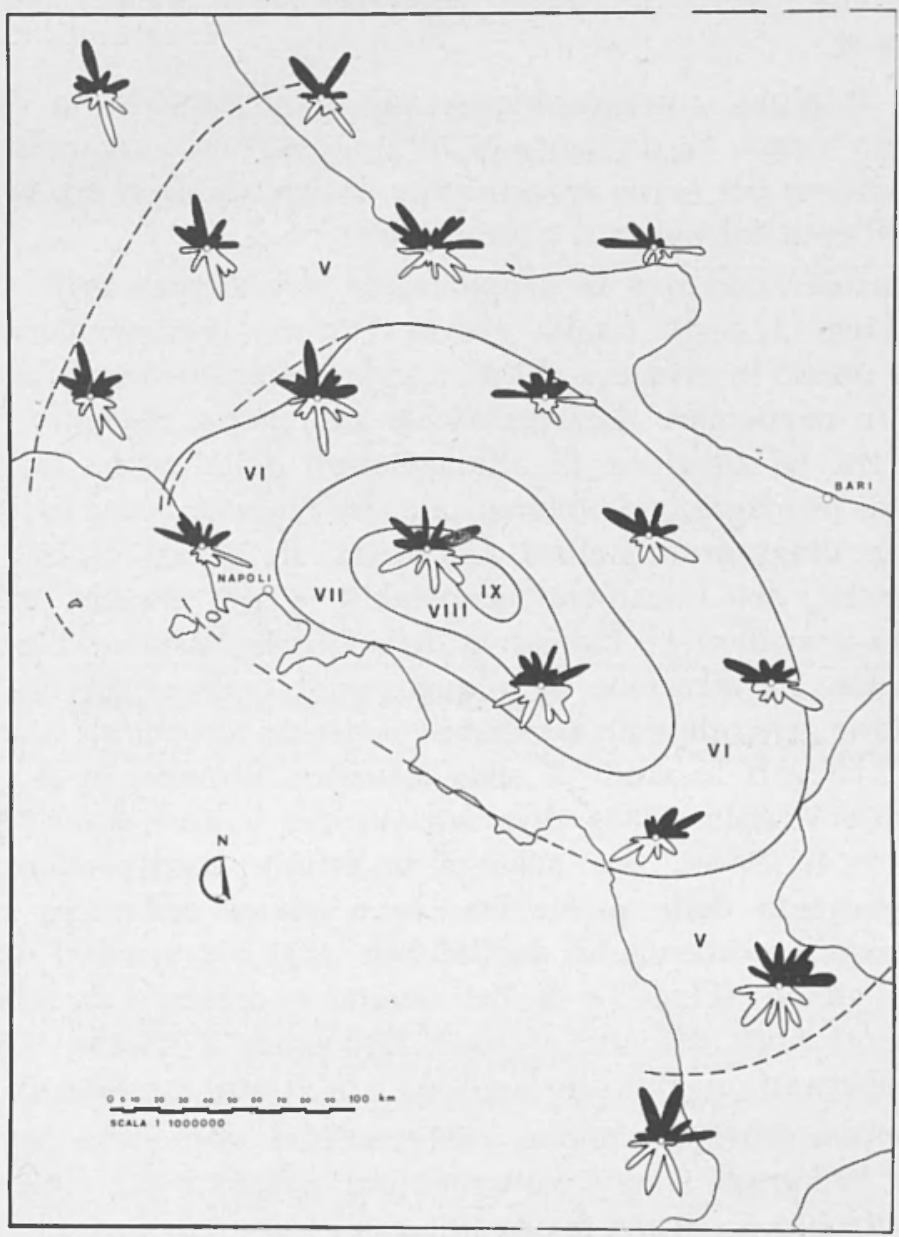

Fig. 4 - Distribuzione dei lineamenti morfologici rilevati con il metodo "Shadow" nell'Italia meridionale e campo regionale del terremoto irpino del 23 Novembre 1980 (particolare). (Da Wise et al., 1979 e da Bottari et al., 1981). Per la interpretazione dei diagrammi vedasi fig. 3 .

trova una buona coerenza con lo sviluppo della penisola salentina (fuori Fig. 4 a SE di Bari).

A Nord di Napoli appare nettamente un massimo con direzione $\mathrm{N} 15^{\circ} \mathrm{W}$. A tale dominio, caratterizzante l'Appennino centrale, 
sono anche riferite localmente numerose faglie normali con direzione N-S.

Il dominio antiappenninico più sviluppato nella regione campano-lucana ha direzione $\mathrm{N} 70^{\circ} \mathrm{E}$ e costituisce un massimo di primo ordine per l'area irpina. Infine il dominio E-W è prevalente solo nell'area del Gargano a NE di Bari.

Considerando ora la distribuzione dei domini nelle singole aree (Figg. 3 e 4), risulta chiara l'ottima corrispondenza con quanto messo in evidenza nel paragrafo precedente circa l'attenuazione. In particolare notiamo che le aree ove si riscontra coincidenza tra la direzione di allungamento della prima isosista e quella di propagazione preferenziale dell'intensità, sono caratterizzate da diagrammi stellari complessi. In questi ultimi, l'alta dispersione dei lineamenti associabili a più domini privi di spiccata prevalenza è indicativa della sovrapposizione di più fasi tettoniche. La direzione di allungamento delle prime isosiste è comunque generalmente associabile a sistemi strutturali recenti ed attuali, rilevati in studi di sismotettonica (BARBANo et al., 1978; GhisetTi e Vezzani, 1982). Diversamente per le aree, come l'Aspromonte e le Serre, ove sussiste un'ottima corrispondenza tra l'allungamento delle prime isosiste e quello dell'intero campo macrosismico, osserviamo domini ben netti e prevalenti nei diagrammi stellari (Figg. 3 e 4). Tali domini - costituiti da sciami di lineamenti poco dispersi - sono associabili a sistemi tettonici attivi, operanti un controllo continuo sull'assetto morfologico.

Da una più approfondita analisi risulta inoltre che la prevalenza e la kurtosi sono i parametri più salienti e più efficaci per tale confronto. In prima approssimazione i domini associati all'andamento preferenziale del campo macrosismico presentano, nel diagramma delle frequenze, valori di prevalenza e kurtosi superiori alla media. Nel diagramma delie lunghezze cumulative invece appaiono massimi di prevalenza e ancor più di kurtosi tanto più alti quanto più accentuato l'andamento anisotropo del campo macrosismico. Le figure 5 e 6 , relative a campi macrosismici dell'Aspromonte (N. 70, Tab. 1) e della Sicilia Nord-orientale (N. 71, Tab. 1) presentano un esempio dei due tipi di correlazione esposta. Nella Tabella 4 sono riportati i valori di prevalenza e di kurtosi per i domini presenti nei relativi diagrammi stellari. 
TABELLA 3

Prevalenza $(P)$ E KURToSi $(K)$ DeI DOMini di Lineamenti NELLA SICILIA NORD-ORIENTALE

\begin{tabular}{r|c|c|c|c}
\hline \multirow{2}{*}{$A_{z}$} & \multicolumn{2}{|c|}{ Numero } & \multicolumn{2}{c}{ Lunghezza cumulativa } \\
\cline { 2 - 5 } & $P$ & $K$ & $P$ & $K$ \\
\hline 78 & 1.29 & 2.53 & 1.40 & 3.29 \\
-11 & 1.17 & 2.04 & 1.08 & 2.90 \\
-72 & 1.07 & 3.36 & 0.95 & 2.90 \\
26 & 0.85 & 2.09 & 0.93 & 2.90 \\
-39 & 0.60 & 1.45 & 0.62 & 1.50 \\
\hline \multicolumn{5}{|c}{} \\
& $\bar{H}=34.46$ & & \\
\end{tabular}

Nota: $A_{z}$, azimut del dominio (rispetto al Nord);

$\bar{H}$ valore medio dell'altezza dei picchi per il calcolo della prevalenza.

TABELLA 3

Prevalenza $(P)$ E KuRtosi $(K)$ DeI Domini Di Lineamenti NELL'AREA DELL'ASPROMONTE (CALABRIA)

\begin{tabular}{r|c|c|c|c}
\hline \multirow{2}{*}{$\Delta_{z}$} & \multicolumn{3}{|c|}{ Numero } & \multicolumn{2}{c}{ Lunghezza cumulativa } \\
\cline { 2 - 5 } & $P$ & $K$ & $P$ & $K$ \\
\hline 36 & 1.97 & 3.35 & 3.17 & 4.27 \\
86 & 0.90 & 1.87 & 0.97 & 1.13 \\
11 & 0.84 & 1.00 & & \\
18 & & & 0.88 & 0.37 \\
-17 & 0.75 & 1.39 & 0.72 & 1.54 \\
-55 & 0.53 & 0.47 & 0.50 & 0.41 \\
54 & & & 0.38 & 0.66 \\
-22 & & & 0.35 & 0.13 \\
\hline
\end{tabular}

$\bar{H}=23.78$

$\bar{H}=13.99$ 


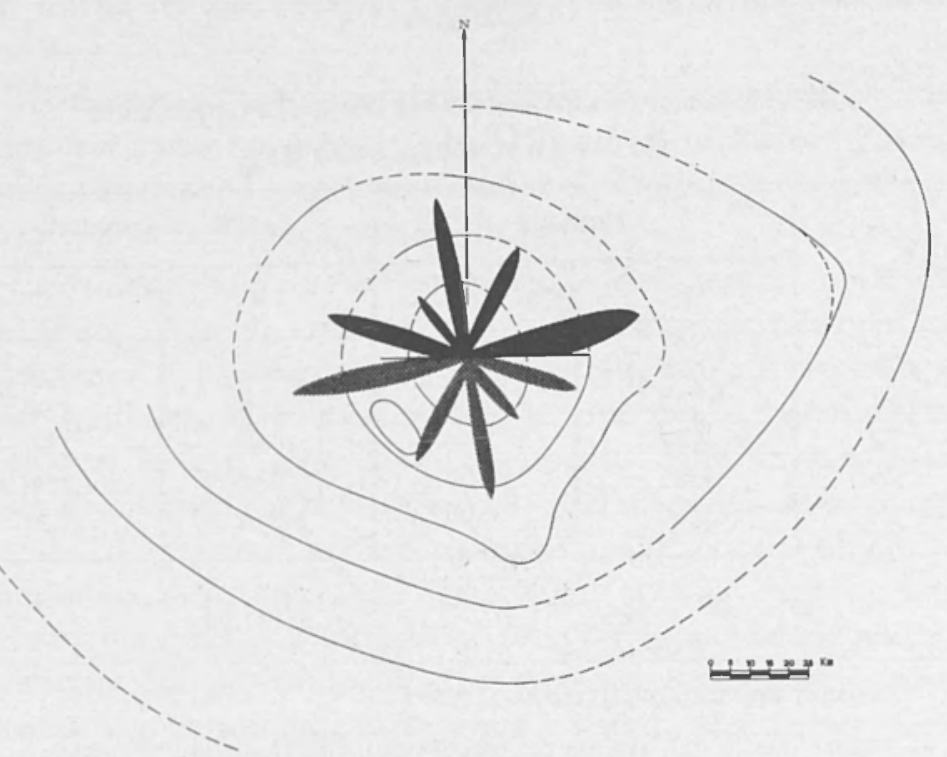

Fig. 5 - Campo macrosismico $\left(I_{o}=8\right)$ del terremoto del Golfo di Patti (N. 71 in Tabella 1) e diagramma dei morfolineamenti "Shadow " per l'area della Sicilia Nord-orientale (N. 1 in Fig. 4).

L'utilizzo del diagramma stellare della «conducibilità » $P_{z}$ consente di visualizzare meglio tale correlazione. La Fig. 7, relativa al terremoto di Ferruzzano ed al campo di lineamenti nell'Aspromonte mostra infatti una netta coincidenza tra i massimi ed i minimi relativi dei due diagrammi stellari. La buona corrispondenza osservabile in questi diagrammi è dovuta in parte alla circostanza che i due campi, macrosismico e strutturale, hanno la medesima estensione e lo stesso centro. Tali condizioni sono da ritenersi ottimali per una più stretta correlazione tra i due campi considerati.

Nel caso di terremoti ad alta energia, i cui campi macrosismici estesi interessano aree con caratteristiche strutturali diverse, la correlazione è più complessa. Un esempio interessante è fornito dal terremoto irpino del 23 Novembre 1980. In tal caso il campo macrosismico si estende dall'Emilia e Romagna alla Sicilia (BotTARI et al., 198l c). Osservando la Fig. 4 si nota che allontanandosi 


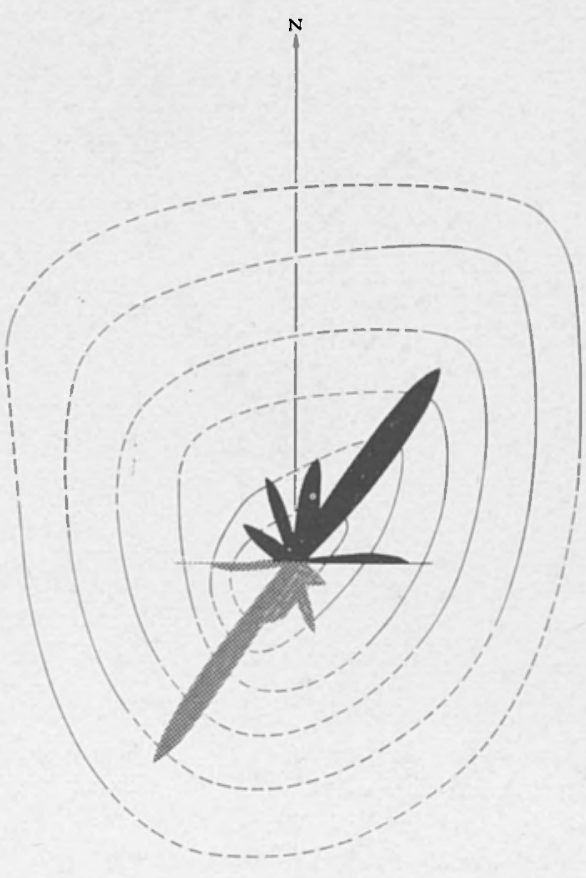

Fig. 6 - Campo macrosismico $\left(I_{o}=7.5\right)$ del terremoto di Reggio Calabria (N. 69 in Tabella 1) e diagramma dei morfolineamenti "Shadow» per l'area dell'Aspromonte (N. 2 in Fig. 4).

dall'area mesosismica la propagazione preferenziale è coerente con i domini di lineamenti prevalenti nelle singole aree. Infatti l'isosista di grado IX risulta più sviluppata lungo la direzione appenninica $\mathrm{N} 45^{\circ} \mathrm{W}$. In tale direzione ma con minore evidenza e lungo la NNW appaiono maggiormente sviluppate le isosiste di VIII e di VII. La direzione di massima propagazione dell'intensità è osservabile fino all'isosista di VI, lungo la dorsale appenninica. A partire da tale isosista, che è l'ultima completa, si comincia ad evidenziare una propagazione preferenziale verso $\mathrm{N} 15^{\circ} \mathrm{W}$ che è rilevabile fino all'isosista di IV. Verso $\mathrm{S}$ la propagazione preferenziale, pur nella incompletezza del tracciato delle isosiste, segue l'andamento dell'Arco calabro-peloritano. 


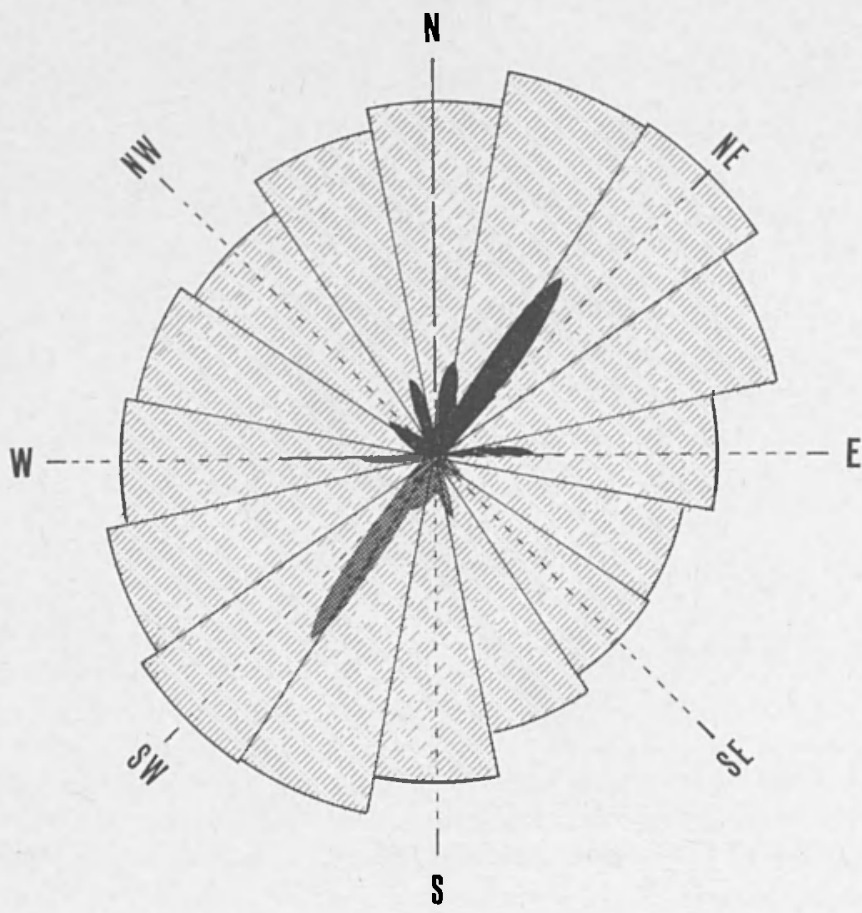

Fig. 7 - Diagramma stellare della conducibilità $P_{z}$ (dal VII al III) per il terremoto di Ferruzzano (N. 70 in Tabella 1) e distribuzione dei morfolineamenti "Shadow" per l'area dell'Aspromonte (N. 2 in Fig. 4). In entrambi i diagrammi $i$ valori sono normalizzati relativamente ai rispettivi valori massimi.

Rileviamo in ultimo la correlazione esistente fra il dominio antiappenninico $\mathrm{N} 70^{\circ} \mathrm{E}$, prevalente nella regione irpina, e la propagazione preferenziale secondaria osservata per questo terremoto e per gli altri citati nel paragrafo precedente in corrispondenza della penisola sorrentina.

\section{Considerazioni CONCLUSIVE}

Le osservazioni esposte e le correlazioni riscontrate sostengono il modello dei Giant Craks formulato da Wise per gli sciami di 


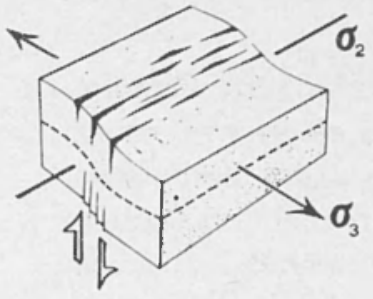

1

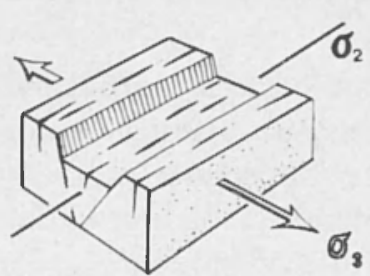

2

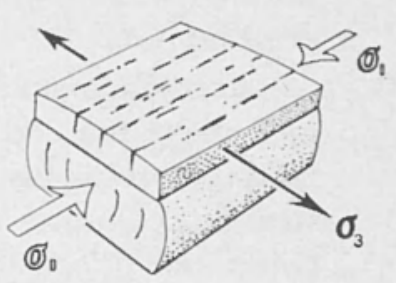

3

Fig. 8 - Alcuni modelli tettonici per la genesi di fasci di lineamenti crostali adottati da Wise et al. (1979).

lineamenti, secondo gli schemi tettonici di Griffith ed Anderson (WISE et al., 1979).

In tale modello i lineamenti morfologici sono tracce in superficie di Cracks intesi come zone di discontinuità piane e subverticali, interessanti la crosta superiore, normali ad un $\sigma_{1}$ regionale orizzontale e subparallele a $\tau_{2}$ e $\sigma_{3}$ (Fig. 8).

In una precedente nota (BotTARI et al., 1981 b) i presenti autori hanno esposto una interpretazione, in chiave sismologica, del suddetto modello. Gli elementi salienti sono riconducibili a: a) anisotropia generata nella parte più superficiale della crosta dagli sciami più densi di lineamenti meno dispersi; b) caratteristiche geometriche degli sciami in relazione alla lunghezza d'onda delle onde trasversali e superficiali. Infatti è ragionevole formulare l'ipotesi che l'anisotropia del campo macrosismico sia determinata dagli sciami più densi di lineamenti e, tra questi, particolarmente da quelli aventi una maggiore lunghezza cumulativa, quindi una maggiore lunghezza media di lineamento ed una più bassa dispersione. Queste due ultime grandezze nel modello esposto, sono indicative rispettivamente: la prima di un maggiore sviluppo verticale delle fratture, la seconda di una genesi dovuta alle fasi tettoniche più recenti. Tale correlazione sembra essere lecita se si pensa che la soluzione di continuità determinata da una frattura recente è senz'altro più netta di quella determinata da una vecchia frattura non rigenerata, totalmente o parzialmente cementata, e che inoltre l'anisotropia generata in un mezzo da un denso sciame 
di fratture è senz'altro maggiore di quella generata da poche grandi faglie.

Considerando il problema in termini più strettamente sismologici rileviamo che l'intensità sismica in un sito esterno al «near field " è principalmente dipendente dalla propagazione delle onde sismiche. Queste, originantisi al fuoco, determinano uno spettro complesso di spostamenti, velocità ed accelerazioni che si modifica con la distanza dalla sorgente in dipendenza dei differenti percorsi, per ciascuna fase sismica, e delle proprietà elastiche delle stratificazioni crostali attraversate. In prima approssimazione è pertanto lecito, su scala regionale, associare la preponderanza degli effetti, nei siti esterni all'area mesosismica, alla propagazione delle onde $S$ e delle onde superficiali. Al transito di queste ultime - tipicamente onde di strato - può attribuirsi il massimo dello scuotimento nelle aree più distanti dal fuoco.

Per quanto rilevato nella precedente nota (BotTari et al., 1981 b) risulta pertanto lecito attribuire grande importanza ai fattori fisici e geometrici che controllano la propagazione delle onde $S$ e delle onde superficiali che, come già sottolineato, sono le principali responsabili delle intensità dello scuotimento in un dato sito. Considerazioni sull'ordine di grandezza della lunghezza d'onda delle onde sismiche e dei parametri geometrici che caratterizzano gli sciami più densi dei lineamenti, consentono di stabilire una stretta correlazione tra "l'ordito", costituito nella parte più superficiale della crosta dai Giant Craks, e le modalità di propagazione dell'intensità. Infatti, ove si considerino le caratteristiche dinamiche dell'onda elastica, è allo sciame di fratture che può attribuirsi il forte assorbimento di energia, allorché la propagazione dell'onda sismica avviene ortogonalmente alla direzione dello sciame medesimo.

Le caratteristiche del campo macrosismico del recente terremoto irpino, cosi come può dedursi dal paragrafo precedente, non solo sostengono questo modello ma, mediante una analisi più approfondita, ne consentono un ulteriore affinamento. A tal fine si rappresenta la funzione $I=I\left(\Delta_{z}\right)$ per le direzioni più salienti del campo in diagrammi semilogaritmici (Fig. 9). Nella Fig. 9a i diagrammi NNW e SW sono relativi rispettivamente alla massima e minima propagazione osservata. Nella Fig. 9 b, sono riportati i 
a)

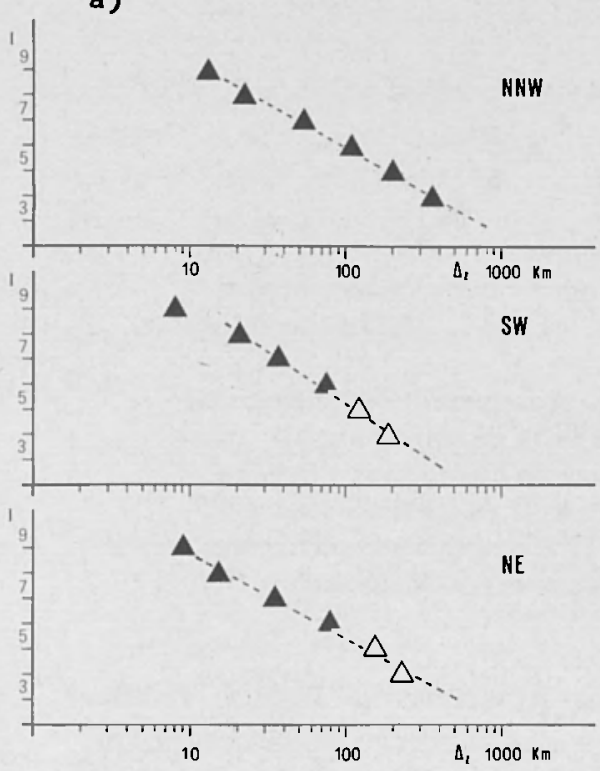

b)

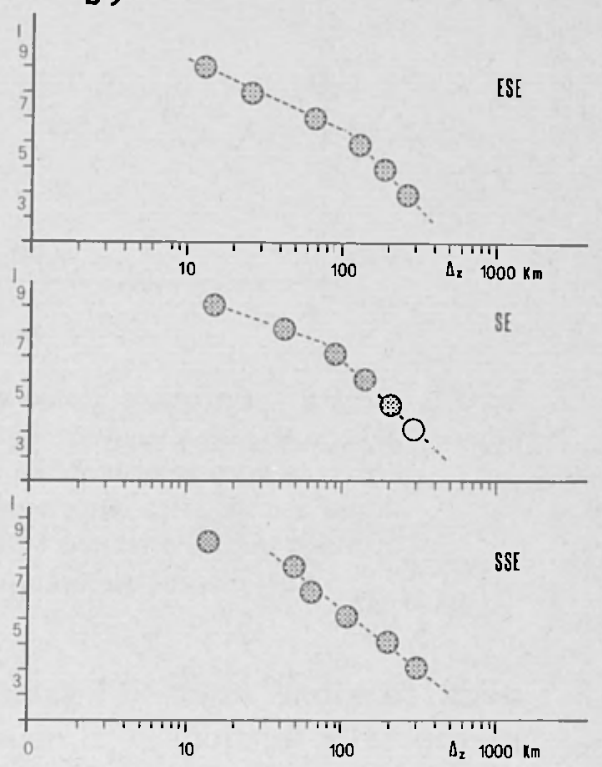

Fig. 9 - Terremoto irpino del 23 Novembre 1980: diagramma dell'intensità sismica in funzione della distanza epicentrale $I=I\left(\Delta_{\mathrm{z}}\right)$, per alcune direttrici salienti del campo macrosismico (i triangoli ed i cerchi vuoti indicano incertezza delle isosiste).

a) lungo queste direttrici l'attenuazione è rappresentabile con un'unica legge esponenziale. La NNW e la SW sono relative rispettivamente alla massima e minima propagazione osservate;

b) questi settori circolari comprendono l'intero arco calabropeloritano. Lungo queste direttrici la funzione non è rappresentabile con una unica legge esponenziale.

diagrammi relativi ai settori angolari (ESE, SE, SSE) comprendenti l'Arco Calabro. In Fig. 9a si nota come l'andamento dell'intensità segue una legge di tipo esponenziale negativo rappresentabile in scala semilogaritmica mediante un unico coefficiente angolare. In Fig. 9b, invece, i diagrammi hanno andamento non riducibile ad un unico coefficiente. Differentemente nel diagramma di Fig. 10, ove in ascissa sono riportati i logaritmi delle distanze misurate lungo l'Arco, si osserva un andamento monotòno 


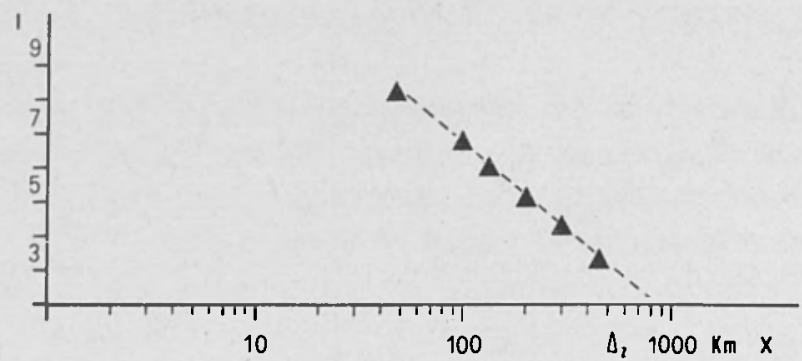

Fig. 10 - Terremoto irpino del 23 Novembre 1980: diagramma della intensità sismica in funzione della distanza misurata lungo l'Arco calabro-peloritano. La funzione in questo caso è rappresentabile con un'unica legge esponenziale. Il valore della conducibilità lungo questa direzione $(416 \div 17)$ è inoltre confrontabile con i valori più alti osservati per l'intero campo macrosismico.

della funzione intensità. Ancor più rimarchevole appare l'andamento della funzione se si considera che il valore della conducibilità $(416 \div 17)$, determinato per la propagazione lungo l'Arco, è confrontabile con i valori più alti osservati nell'intero campo (vedi Tab. 1). Pertanto, quanto esposto consente un ulteriore verifica ed affinamento del modello poiché si constata che "l'ordito" degli sciami di fratture, più densi e ben sviluppati nelle singole aree, non solo controlla l'attenuazione bensì sembra avere funzione di guida per le onde superficiali. 


\section{BIBLIOGRAFIA}

Baratta, M., 1901 - I terremoti d'Italia, ed. Bocca, Torino.

Barbano, S.M., Bottari, A., Carveni, P., Cosentino, M., Federico, B., Fonte G., Lo Giudice, E., Lombardo, G., PatanE, G., 1979 - Macroseismic study of the Gulf of Patti earthquake in the geostructural frame of northeastern Sicily. "Boll. Soc. Geol. Ital.", 98, pp. 155-174.

Barbano, S.M., Carrozzo, M.T., Carveni, P., Cosentino, M., Fonte, G., Ghisetti, , F., Lanzafame, G., Lombardo, G., Patané, G., Ruuscetti, M., Tortorici, L., Vezzani, L., 1978 - Elementi per una carta sismotettonica della Sicilia e della Calabria meridionale. "Mem. Soc. Geol. Ital.", XIX, pp. 625-632.

BLAKE, A., 1941 - On the estimation of focal depth from macroseismic data. "Bull. Seism. Soc. Am.", 31, p. 3.

Bottari, A., Federico, B., Lo GiUdice,E., 1979 - Methodological considerations regarding the determination of some macroseismic field parameters. Application to Earthquake in the Calabro-Peloritan Arc. "Boll. Geofis. Teorica ed Applicata", 21, pp. 197-225.

Bottari, A., Lo Giudice, E., Nicoletti, P., Sorriso-Valvo, M., 1981 - The Ferruzzano earthquake of 1978: macroseismic effects and slope-stability conditions in Southern Calabria (Italy). "Rev. Geogr. Phys. Geol. Dynam.", 23, pp. 73-84.

Bottari, A., Federico, B., Lo Giudice, E., $1981 \mathrm{~b}$ - On the correlation between macroseismic attenuation trend and geo-structural framework for CalabroPeloritan Arc. "Tectonophysics" in press).

Bottari, A., Federico, B., Giovani, L., Lo Giudice, E., Spadea M.C., Vecchi, M., 1981 - Il terremoto irpino del 23 Novembre 1980: il campo macrosismico e lattenuazione dell'intensità. Atti Convegno Sismicità dell'Italia, C.N.R., PF Geodinamica, "Rend. Soc. Geol. It.", pp. 459-552.

Branno, A., Esposito, E.G.I., Marturano, A., Porfido S., Rinaldis, V., 1982 - $l l$ terremoto della Basilicata del 16 Dicembre 1857.

C APUTO, M., PostPISCHL, D., 1974 - Countour mapping of seismic areas by numerical filtering and geological implications. "Annali di Geofisica", 27, pp. 619-639.

GHISETTI, F., VEZZANI, L., 1979 - Quadro neotettonico preliminare della Sicilia orientale, nuovi contributi alla realizzazione della carta neotettonica d'Italia. Pubbl. N. 251, pp. 333-340, Italian Geodynamical Project.

GhisetTI, F., VezZANI, L., 1982 - Different styles of deformation in the Calabrian Arc (Southern Italy): implications for a seismotectonic zoning. "Tectonophysics", 85 , pp. 149-165. 
Lo GiUdice, E., PATANE, G., RaSA, R., Romolo, R., 1982 - The structural framework of Mt. Etna. "Mem. Soc. Geol. It.", XXIII, pp. 125-158.

Marcelli, L., S PAdEA, M.C., VeCCHI, M., 1979 - Considerazioni sul problema della interpretazione ed elaborazione di dati macrosismici. C.N.R. PFG N. 238.

Ogniben, L., Parotto, M., Praturlon, A. (Editors), 1975 - Structural model of Italy. Quaderni della Ricerca Scientifica N. 90, C.N.R., p. 502.

SERVA, L., 1981 a - Il terremoto del 1688 nel Sannio, in contributo alla caratterizzazione della sismicità del territorio italiano. Comm. ENEA-ENEL, Convegno C.N.R. PF Geodinamica, pp. 209-250, Udine 1981.

Serva, L., 1981 b - Il terremoto del 1964 in Irpinia-Basilicata. Comm. ENEA-ENEL, Convegno C.N.R. PF Geodinamica, pp. 183-208, Udine 1981.

SHEBALIN, N.V., 1972 - Macroseismic data as information on source parameters of large earthquakes. "Phys. Earth. Planet. Interior", 6, pp. 316-323.

Shebalin, N.V., 1974a - Principles and procedures of cataloguing, catalogue of earthquakes in the Balcan Region. UNESCO, Skopje.

Shebalin, N.V., 1974b - Foci of strong earhquakes in the USSR (in Russian). Publ. "Inst. of Phys. Earth Lenin", USSR Academy of Science, Moscow.

SPONHEUER, W., 1960 - Methoden zur herdtiefenbestimmung in der makroseismik. "Freiberger Forschungshefte", C 80, Berlin.

WISE, D.U., 1969 - Pseudo-radar topographic shadowing for detection of sub-continent sized fracture systems. "Proceeding of the Sixth International Symposium in the Remote Sensing of Environment", Univ. of Michigan, pp. 603-615.

Wise, D.U., Funicello, R., Parotto, M., SalvinI, F., 1979 - Domini di lineamenti e di fratture in Italia. Pubbl. dell' 'Istituto di Geologia » dell'Università di Roma. 